
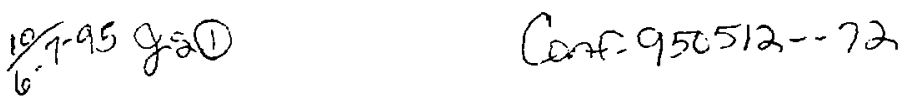

SLAC-PLB-95-6903

May 1995

\title{
Nonlinear Analyses of Storage Ring Lattices Using One-Turn Maps *
}

\author{
Y.T. Yan, J. Irwin, and T. Chen \\ Stanford Linear Accelerator Center, Stanford University, Stanford, CA 94309 USA
}

\begin{abstract}
Abstracl
Using normalized one-turn resonance-basis Lie generators in conjunction with an action-angle tracking algorithm (nPB tracking), we have been able to better understand the relationship between the dynamic aperture and lattice nonlinearities. Tunes, tune shifts with amplitude and/or energy, and resonance strengths may be freely changed to probe their individual impact on the dynamic aperture. Fast beam-beam simulations can be performed with the inclusion of nonlinear lattice effects. Examples from studies of the PEP-II lattices are given.
\end{abstract}

\section{INTRODUCTION}

Simple lattice elenent-by-element tracking for $d y$ narnic aperture determination is essential but limited by the fact that information is obtained at only one working point and one set of lattice parameters. Furthermore, inadvertent errors in the lattice and control files can remain undetected. To enhance our understanding of lattice monlinearities and their relationship with the dynamic aperture, we have developed a set of one-turn mapping procedures that allow us to obtain one-turn resonance-basis Lit generators for circular accelerator nonlinear lattice studies.

Contained in the Lie generators are tune-shift and resonance terms of different orders. These terms can be suitably normalized far comparisons among themselves or with those obtained from one of a series of lattices that are under improvement. Furthermore, by directly taking Poisson bracket expansion of the resonance-basis Lie generators to a suitable order to evaluate the turn-by-turn Lie transformations, one not only can achieve a very fast tracking for dynamic aperture determination to obtain swamp plots (dynamic aperture vs. tune), but also can freely change selected tune-shift or resonance terms to probe their individual impact on the dynamic aperture.

In the following sections these one-turn mapping procedures are described and examples for their applications in PEP-II lattice development are presented.

\section{The One-Turn Resonance Basis Map}

To obtain resonance basis map for a lattice we first extract a one-turn map at a suitable observation position as a Taylor expansion about the on-momentum closed orbit. In general, we include all lattice nonlinearities. However, we can concentrate on a particular lattice module by inserting a linear lattice for the rest of the ring. We usually consider 2-dimensional maps with a parameter $\delta$ representing the momentum deviation $\mathrm{dp} / \mathrm{p}$. Thus, the Taylor map can be expressed as

-Work supported by the Department of Energy under Cantract No. DE-AC03-76SFOO515

$$
\vec{z}=\bar{U}(\bar{z}, \delta)+\emptyset(N+1),
$$

where $\emptyset(N+1)$ indicates that the Taylor map is truncated at an order of $N, \vec{z}=\left(x, p_{x}, y, p_{y}\right)$ is the global or initial phase-space coordinate vector and $\vec{Z}=\left(X, P_{x}, Y, P_{y}\right)$ is the phase-space coordinate vector after one turn.

Once the one-turn Taylor map is obtained, we make a Floquet transformation such that

$$
\bar{z}=\mathcal{A}^{-1}(\vec{z}, \delta) \mathcal{R}(\vec{z}) e^{: \int(\vec{z}, \delta)} \cdot \mathcal{A}(\vec{z}, \delta) \vec{z}+\emptyset^{\prime}(N+1),
$$

where $\mathcal{R}(\bar{z})$ is one-turn pure rotational map in the 4 dimensional transverse canonical phase-space, and $\mathcal{A}(\vec{z}, \delta)$ and its inverse $\mathcal{A}^{-1}(\vec{z}, \delta)$ are the $4-\mathrm{by}-5$ matrices that generate the Floquet transformation. The dispersion, $\eta$, and the Courant-Snyder parameters, $\alpha, \beta$, and $\gamma$ are all included in $\mathcal{A}(\vec{z}, \delta)$ and $\mathcal{A}^{-1}(\bar{z}, \delta)$. Making the Floquet transformation $\bar{z}_{F}=\mathcal{A}^{-1}(\vec{z}, \delta) \vec{z}$ and then dropping the subscript $F$ for convenience, one obtains a one-turn map

$$
\vec{z}=R(z) e^{S(\vec{z}, \delta)} \bar{z} .
$$

The polynomial $f(\vec{z}, \delta)$ of the Lie transformation in Eq. 3 can be decomposed in a complete basis consisting of the rotational eigen-modes, $\dot{x}_{ \pm}=x \mp i p_{x}=$ $\sqrt{2 J_{x}} e^{ \pm i \theta_{z}}, \hat{y}_{ \pm}=x \mp i p_{y}=\sqrt{2 J_{y}} e^{ \pm i \theta_{x}}$, where $J_{x}, J_{y}, \theta_{x}$, and $\theta_{y}$ are action-angle variables. One then obtains

$$
\begin{aligned}
& f(\vec{z}, \delta)=
\end{aligned}
$$

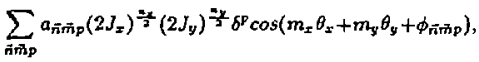

where the terms with $m_{x}=m_{y}=0$ are the tune shift terms [1]. For convenience, all these tune shift ierms are grouped together and represented by $h_{T}\left(J_{x}, J_{y}, \delta\right)$. The remaining terms, all with angular variable dependence, are also grouped and represented by $h_{R}\left(J_{x}, J_{y}, \theta_{x}, \theta_{y}, \delta\right)$. Thus, the one-tum map given by Eq. 3 can be written as

$$
\vec{Z}=e^{:-\mu_{x} J_{x}-\mu_{y} J_{y}} e^{i-h_{T}\left(J_{x}, J_{y}, d\right)-h_{R}\left(\partial_{x}, J_{x}, \partial_{y}, J_{y}, \delta\right) z_{1}}
$$

where we have replaced the rotation $\mathcal{R}(\hat{z})$ with its Lie representation $e^{:-\mu_{x} J_{x}-\mu_{y} J_{y}}$, where $\mu_{x}$ and $\mu_{y}$ are the working tunes of the lattice. This is the resonance basis map.

\section{Iil. NORMALIZATION OF TUNE SHIFT AND RESONANCE COEFFICIENTS}

It should be noted that $h_{T}, h_{R}$, and the action coordinates $\left(J_{x}, J_{y}\right)$ in Eq, 5 have the dimensions of emittance while $\theta_{x}, \theta_{y}$, and $\delta$ are dimensionless. Therefore, the coeffcients in the polynomials $h_{T}$ and $h_{R}$ have different dimensions. For convenience in directly using these coefficients 
for calculating and comparing the tune shift and resonance strength of different orders, we introduce a scaling transformation such that $h_{T}=\epsilon_{x} \hat{h}_{T}, h_{R}=\epsilon_{x} \hat{h}_{R}, J_{x}=\epsilon_{x} \hat{J}_{T}$, and $J_{y}=\epsilon_{x} \hat{J}_{y}$ to obtain the dimensionless one-turn map which, after dropping the symbol; is again given by Eq. 5 except with modified coefficient values. Note that $\epsilon_{x}$ is the horizontal emittance, which in PEP-11 is $48 \mathrm{~nm}$-rad for the High-Energy Ring (HEP) and $64 \mathrm{~nm}$-rad for the the LowEnergy Ring (LER).

In our numerical studies for PEP-I] lattices, we set $\epsilon_{y}=\frac{1}{2} \epsilon_{x}$ to obtain the required vertical aperture that is sufficient for injection and for vertical blow-up from the beam-beam interaction. Most often we calculate the resonance strength and tune shift along the 100 (10 times the nominal beam size) ellipse $r_{x}^{2}+\frac{s_{x}}{c_{x}} r_{y}^{2}=N^{2}$ with $\frac{s_{x}}{s_{y}}=2$ and $N=10$, where $r_{x}=\sqrt{2 J_{x}}$, and $r_{y}=\sqrt{2 J_{y}}$ are radii in the two-dimensional pbase-space planes.

\section{A. TUNE SHIFT}

Using Hamilton's equations and the effective Hamiltonian $h_{T}$ in Eq. 5, one can obtain both horizontal $(x)$ and vertical $(y)$ tune shitts as explicit polynomials in the geometric invariants $J_{x}$ and $J_{y}$ and the chromatic amplitude $\delta$, given by

$$
\Delta \nu_{x}\left(J_{s}, J_{y}, \delta\right)=\frac{1}{2 \pi} \frac{\partial h_{T}\left(J_{x}, J_{y}, \delta\right)}{\partial J_{x}}
$$

and

$$
\Delta \nu_{y}\left(J_{x}, J_{y}, \delta\right)=\frac{1}{2 \pi} \frac{\partial h_{T}\left(J_{x}, J_{y}, \delta\right)}{\partial J_{y}} .
$$

To make comparison of tupe shift terms of different order, we usually calculate the maximum of each term along the $10 \sigma$ ellipse.

\section{B. RESONANCES}

Since resonance terms (in $h_{R}$ ) of higher orders have larger derivatives, thereby causing larger step-sizes in phase space, we prefer to measure the strength of a resonance term by taking its Poisson bracket (PB) with respect to phase space coordinates $J_{x}, J_{y}, \theta_{x}$, and $\theta_{y}$. From these PBs we compute the phase-space step [2]

$$
|\Delta z|=\sqrt{\left[\left(r_{z} \Delta \theta_{x}\right)^{2}+\left(\Delta r_{x}\right)^{2}\right]+\frac{\epsilon_{x}}{\epsilon_{y}}\left[\left(r_{y} \Delta \theta_{y}\right)^{2}+\left(\Delta r_{y}\right)^{2}\right]} .
$$

We then compute the maximum value of $|\Delta \bar{z}|$ for all possible values of $\theta_{x}, \theta_{y}, J_{x}$, and $J_{y}$ with the constraint $r_{x}^{2}+c_{x} r_{y}^{2}=N^{2}$. This maximum is what we call the norugalized resonance basis coefficient. $\mid \Delta=1=1$ means that the corresponding resonance can at most cause a phasespace motion of $1 \sigma$ in one turn for a particle on the $10 \sigma$ boundary.

\section{A SAMPLE PLOT}

Each of the tune shift and resonance terms is uniquely represented by a set of indices $(\vec{n}, \vec{m}, p)$. For a map of $10^{\text {th }}$. order, there would be thousands of terms. Although most of the terms are essential to the lattice nonlinear behavior, in search for improvement of the lattice, one only needs to pay attention to a limited number of larger terms. As an example, Figure 1 shows the normalized tune shift and resonance coeficients that are larger than 0.01 for a PEPII LER bare lattice.

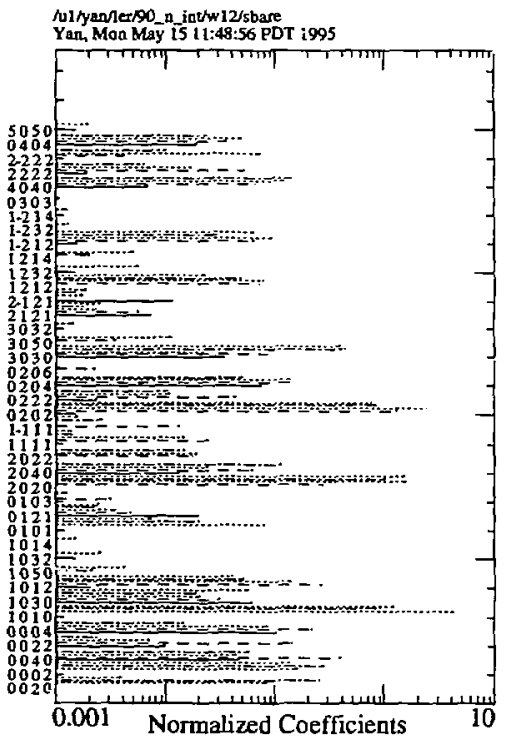

Figure. !. Normalized tune shift and resonance coefficients plotted in log seale horizontally. The vertical axis shows corresponding indices $\left(m_{x}, m_{y}, n_{x}, n_{y}\right)$ for resonances and orders. The corresponding chromatic indices, $p$ 's, are not explicitly stown in the axis but are indicated with line patterns ( $\mathrm{p}=0$ : solid, 1: dashes, 2: dots, 3: dotdashes, etc.

\section{IV. nPB TRACKING AND ITS RELIABILITY}

The normalized tune shift and resonance coefficients described in the last section can help us indentify a limited number of terms that would degrade the dynamic aperture. To understand deeper and confirm more precisely their individual impacts on the dynamic aperture, we can freely change the corresponding coefficients and then evaluate the updated resonance basis map to see the change of the dynamic aperture.

To evaluate a resonance basis map, we directly take Poisson bracket expansion of the resonance basis Lie generators to a suitable (n) vider and so the name of $\mathrm{DPB}$ track- 
ing. The procedure of $\mathrm{nPB}$ tracking is basically to perform turn-by-turn tracking of the particle phase-space coordinates. This is done by evaluating the one-turn map given by Eq. 2 followed by an update of the particle momentum deviation $(\delta)$ through an accurate but concise time-of-light map. Note that in evaluating the Lie transformation, the Lie generator, $f=-h_{T}-h_{R}$, is kept in the action-angle variable space while the particle phaserspace coordinates ase always kept in Cartesian coordinates which are considered as functions of the aetion-angle variables for the Paisson bracket calculation - this is the key to the fast computational speed of the nPB tracking since all the Sines and Cosines can he calculated only once and stored for repeated turn-by-turn tracking [3].

As to the reliability of the $\mathrm{DPB}$ tracking, one may be concerned with the fact that the nPB tracking is not $100 \%$ accurate since the map is truncated at a moderate order and not $100 \%$ symplectic since one does not carry the Poisson bracket expansion to the infinite order. However, it is well understoud that the required accuracy and symplecticity depend on circumstances [4]. For the PEP-II lattice dyaamic aperture studies (only 1024 turns needed because of synchrotron radiation damping), from sumerous tests we have concluded that a $10^{\text {th }}$-order map with 3-Poisson-bracket expansion of the Lie transformation is accurate and symplectic enough. It takes about 1 minute with such a $10^{\text {th }}$-order map, 3PB tracking on a RISC workstation to obtain a dynamic aperture plot at a given working point, which would otherwise have taken a few hours with element-by-element tracking.

\section{SWAMP PLOTS FROM nPB TRACKING}

The fast computational speed of nPB tracking allows fast calculation of dynamic aperture and so one can obtain a swamp plot for a given lattice in a reasonable time. Tc obtain a swamp plot with the $\mathrm{DPB}$ tracking, one would follow exactly the nPB tracl:ig procedures described in Section IV, except that one would increment the working tunes $\mu_{x}$ and $\mu_{y}$, while keeping all other terms in the resonance bzsis map fixed, to obtain dynamic apertures throughout the tune plane. This is equivalent to using element-by-element tracking and inserting an exactly makched linear tromhone to switch the working tunes without further changing the lattice. In our practice, we have generally found such swamp plots very informative. They have helped us in evaluating and improving the PEP-II lattices. Occasionally we would check a few spots of a swamp plot against corresponding element-by-element irackings to ensure that there are no surprises.

Some typical PEP-II lattice swamp plots can be found in Ref. [5].

\section{BEAM-BEAM WITH nPB TRACKINGS}

The fast speed of the nPB tracking allows one to in. clude the arc lattice as a nonlinear resonance-basis map for beam-beam simulations. To further enhance the tracking speed, one can even drop irrelevant resonance terms. As an example, shown in Figure 2 are the beam tail distributions of the PEP-II HER $\beta_{y}^{*}=2.0 \mathrm{~cm}$ lattice with and without nonlinear terms in the one-turn map.
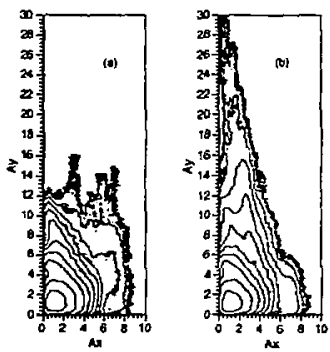

Figure. 2. The beam tail distribution of PEP-1I HER: (a) with linear lattice, and (b) additionally including tuneshift-with-amplitude terms.

\section{SUMMARY}

The one-turn mapping procedures described above have been important for PEP-II lartice development. During the course of numerous PEP-II lattice updates, we were able to identify important tune shiti and resonance terms that would degrade the dynamic aperture. We then confirmed and understood their individual impacts on the dynamic aperture with $\mathrm{nPB}$ tracking and swamp plots. thereby improving the lattice.

\section{ACKNOWLEDGEMENT}

One of the aurhors (YTY) thanks John Seeman for his patience in waiting for the development of these non-lipear analysis tools for PEP-II lattice studies.

\section{References}

[1] For more accurate une shift calculation, onc should use nonlinear normal form.

[2] J. Irwin, N. Walker, and Y. Yan, "The Application of Lie Algehra Methods in the PEP-1l Design," SLACPUB-95-6779, in Proc. of the 4th EPAC, p. 899 (1994).

[3] J. Irwin, T. Chen, and Y. Yan, "A fast tracking method using resonance basis Hiımiltonians," SLAC-PBL-956727.

[4] Y.T. Yan, "Application of differential algebra to singleparticle dynamics in storage rings", in Physics of Purticle Accelerators, AIP Conf. Proc. No. 249, edited by M. Month, and M. Diene (AIP, New York, 1992), p. 378.

[5] Y.T. Yan, at al., "Swamp Plots for Dynamic Aperture Studies of PEP-11 Lattices," SLAC-PUB-95-6876, also in these proceedings. 


\section{DISCLAIMER}

This report was prepared as an account of work sponsored by an agency of the United States Government. Neither the United States Government nor any agency thereof, nor any of their employees, makes any warranty, express or implied, or assumes any legal liability or responsibility for the accuracy, completeness, or usefulness of any information, apparatus, product, or process disclosed, of represents that its use would not infringe privately owned rights. Reference herein to any specific commercial product, process, or service by trade name, tradernark, manufacturef, or otherwise does not necessarily constitute or imply its endorsement, recommendation, of favoring by the United States Government or any agency thereof. The views and opinions of authors expressed herein do not necessarily state or reflect those of the United States Government or any agency thercof. 